\title{
New diabetes drugs go beyond insulin to flush out excess sugar
}

The list of approved medications for type 2 diabetes is long and varied. Some drugs come in pill form; others must be injected. Some cause weight gain; others trigger weight loss. But all existing treatments to lower blood sugar, different as they may be, essentially work by modulating the activity of insulin. These drugs have proven quite effective in the short term, but they tend to lose their potency over time. As a result, drug companies have been racing to develop new diabetes medicines that act on a pathway that doesn't involve insulin.

A drug called dapagliflozin could be the first such agent to gain market approval. Co-developed by Bristol-Myers Squibb (BMS) and AstraZeneca, dapagliflozin reduces blood glucose levels in an insulin-independent manner by preventing a protein called sodium-dependent glucose cotransporter 2 (SGLT2) from reabsorbing glucose in the kidney. As such, the simple sugar gets excreted through the urine instead of reentering the blood stream and elevating blood sugar levels, a condition known as hyperglycemia, one of the main symptoms of diabetes.

Dapagliflozin is "intriguing in that the mechanism is different," says Edward Chao, an endocrinologist at the University of California-San Diego Medical Center, who is not affiliated with any drug companies developing SGLT2 inhibitors. "No medicine for diabetes has used the kidney as a means to help control sugar."

Earlier this year, off the back of 40 clinical trials testing dapagliflozin in around 6,000 people, the two drug giants filed for approval with the US Food and Drug Administration. But dapagliflozin is not the only medicine in its class nearing regulatory approval. Johnson \& Johnson is currently conducting a twoyear, multicenter phase 3 trial evaluating the ability of its SGLT2 inhibitor, called canagliflozin, to reduce both blood glucose and body weight. Meanwhile, Boehringer Ingelheim and Lexicon Pharmaceuticals have their own SGLT2-blocking compounds in phase 2 testing, and other companies have drugs earlier in development.

Richard Kibbey, an endocrinologist at the Yale University School of Medicine in New Haven, Connecticut, welcomes the development of all these new SGLT2 inhibitors. Drugs that "let the body control glucose metabolism itself [are] probably the best way to control diabetes," he says.

But SGLT2 inhibitors can do more for diabetics than just lower blood glucose concentrations. In a meta-analysis published in April, researchers at the University of Turin in Italy surveyed 13 placebo-controlled studies testing four different companies' compounds and found that people taking the drugs also experienced weight loss and had lower blood pressure than those on placebo pills (Ann. Med. doi:10.3109/07853 890.2011.560181, 2011). The same month, BMS scientists presented new clinical data at the American Association of Clinical Endocrinologists annual meeting in San Diego showing that dapagliflozin either alone or together with the antidiabetic medicine metformin also improves the function of the pancreas's insulin-secreting beta cells, which are often damaged by excess glucose concentrations in people with type 2 diabetes. What's more, preliminary data from the various companies' clinical trials indicate that the drugs help reduce high blood triglycerides and lower cholesterol, too, according to Niloofar Tabatabai, a molecular physiologist who studies glucose transport at the Medical College of Wisconsin in Milwaukee.

Beyond type 2 diabetes, many researchers are also confident that the same drugs could be effective in treating the autoimmune form of the disease, known as type 1 or juvenileonset diabetes. However, Kibbey notes that the drugs would probably need to be combined with insulin injections, as people with type 1 diabetes typically fail to produce insulin entirely rather than just have trouble regulating the enzyme's activity, as happens in people with the adult-onset form of the disease.

Monica Heger

\section{Disputed EU herbal medicine rules take force}

A controversial set of rules in the EU that changes the way herbal medicinal products are licensed and marketed in Europe came into full force this May, ending a seven-year grace period that had allowed manufacturers to come up to speed on the new measures.

Under the law, any plant product with a new curative claim sold over the counter is subject to an approval process the same as the one that pharmaceutical drugs are required to undergo when seeking marketing authorization. Additionally the new regulations, known as the Traditional Herbal Medicinal Products Directive, will force natural products producers to adhere to the standards of good manufacturing practice, a set of guidelines designed to ensure the quality of a product.

Although the use of herbal medicines in Europe is on the rise, the safety regulations governing these products in the $\mathrm{EU}$ have been "a mess," according to Frederic Vincent, spokesperson for the EU Directorate General for Health and Consumers in Brussels.

There has been a considerable public outcry within the European community. A petition led by the web-based political action group Avaaz aims to amend this legislation in Brussels to remove all barriers to traditional remedies with a long history of use inside and outside of Europe. Opponents fear it will lead to a reduction in the choice of herbal remedies, leaving big pharma with a monopoly on natural medicinal product development and smaller manufacturers traditionally in the business unable to come up with the increased licensing costs.

The petition might be seen by some as futile, however, because the law has already been implemented at national levels. And there has been an ongoing erosion occurring for several decades that has gone largely unnoticed even by many medical practitioners, with traditional herbal products steadily disappearing from shelves.

"On the one hand, this is a good law. We want regulation," says Michael Hartmann, a hospital pharmacologist in Jena, Germany and an advisor on medicinal products for the European Council. "But this will most certainly restrict thousands of traditional Chinese medicinal products that have not yet been registered in the EU as well as any combinations of vitamins and plant products from entering the market," he says.

Ayurvedic and traditional Chinese medicinal products are often plant mixtures that until recently have been available as food supplements and must now be registered as medicinal products.

Karen Dente 